\title{
Identification of novel diphenyl urea inhibitors of Mt-GuaB2 active against Mycobacterium tuberculosis
}

\author{
Veeraraghavan Usha, ${ }^{1}$ Sudagar S. Gurcha, ${ }^{1}$ Andrew L. Lovering, ${ }^{1}$ \\ Adrian J. Lloyd, ${ }^{2}$ Athina Papaemmanouil, ${ }^{1}$ Robert C. Reynolds ${ }^{3}$ \\ and Gurdyal S. Besra ${ }^{1}$ \\ ${ }^{1}$ School of Biosciences, University of Birmingham, Edgbaston, Birmingham B15 2TT, UK \\ ${ }^{2}$ Department of Biological Sciences, University of Warwick, Coventry, UK \\ ${ }^{3}$ Drug Discovery Division, Southern Research Institute, Birmingham, AL 35255, USA
}

Correspondence

Gurdyal S. Besra

g.besra@bham.ac.uk

Received 11 June 2010

Revised 13 October 2010

Accepted 21 October 2010

\begin{abstract}
In contrast with most bacteria, which harbour a single inosine monophosphate dehydrogenase (IMPDH) gene, the genomic sequence of Mycobacterium tuberculosis H37Rv predicts three genes encoding IMPDH: guaB1, guaB2 and guaB3. These three genes were cloned and expressed in Escherichia coli to evaluate functional IMPDH activity. Purified recombinant Mt-GuaB2, which uses inosine monophosphate as a substrate, was identified as the only active GuaB orthologue in M. tuberculosis and showed optimal activity at $\mathrm{pH} 8.5$ and $37^{\circ} \mathrm{C}$. Mt-GuaB2 was inhibited significantly in vitro by a panel of diphenyl urea-based derivatives, which were also potent anti-mycobacterial agents against $M$. tuberculosis and Mycobacterium smegmatis, with MICs in the range of $0.2-0.5 \mu \mathrm{g} \mathrm{ml}^{-1}$. When Mt-GuaB2 was overexpressed on a plasmid in trans in M. smegmatis, a diphenyl urea analogue showed a 16-fold increase in MIC. Interestingly, when Mt-GuaB orthologues (Mt-GuaB1 and 3) were also overexpressed on a plasmid in trans in $M$. smegmatis, they also conferred resistance, suggesting that although these Mt-GuaB orthologues were inactive in vitro, they presumably titrate the effect of the inhibitory properties of the active compounds in vivo.
\end{abstract}

\section{INTRODUCTION}

Every year, 9 million people are newly diagnosed with tuberculosis (TB) and there are about two million TB deaths each year (Corbett et al., 2003). Furthermore, increased incidences of multi-drug resistant (MDR)-TB and extensively drug resistant (XDR)-TB strains have caused an alarming rise in the number of $\mathrm{TB}$ cases (Kaufmann \& Parida, 2007). Since effective new drugs are urgently needed for the treatment of MDR-TB, XDR-TB and HIV-associated TB cases, there is a need to identify and characterize potential new drug targets.

Inosine monophosphate (IMP) is synthesized via several steps from phosphoribosyl pyrophosphate. IMP is a common metabolic precursor for both adenine and guanine nucleotides. Guanosine monophosphate (GMP) is synthesized in two stages from IMP (Lambden \&

Abbreviations: DPU, diphenyl urea; GMP, guanosine monophosphate; IMP, inosine monophosphate; IMDPH, IMP dehydrogenase; MDR, multidrug resistant; TB, tuberculosis; XDR, extensively drug resistant; XMP, xanthosine monophosphate.

Two supplementary tables of primer sequences are available with the online version of this paper.
Drabble, 1973). The first step of GMP synthesis involves the conversion of IMP to xanthosine monophosphate (XMP) and is performed by inosine monophosphate dehydrogenase (IMPDH, IMP-NAD ${ }^{+}$oxidoreductase, E.C. 1.1.1.205) with concomitant conversion of $\mathrm{NAD}^{+}$to $\mathrm{NADH}$, as shown in Fig. 1(a) (Jackson et al., 1975). XMP is then subsequently converted to GMP by a GMP synthetase (Gilbert et al., 1979). IMPDH catalyses these two very different chemical transformations, firstly a dehydrogenase reaction to form $\mathrm{NADH}$ and the covalent enzyme intermediate $\mathrm{E}-\mathrm{XMP}^{\star}$, and secondly a hydrolysis reaction, which converts E-XMP* into XMP (Hedstrom, 2009).

IMPDH is a rate-limiting enzyme in guanine nucleotide metabolism and inhibition of IMPDH depletes cellular guanine nucleotides (Hedstrom, 2009; Pimkin et al., 2009). The IMPDH reaction was first reported in 1957 in extracts of Aerobacter aerogenes (Magasanik et al., 1957). To date, IMPDH enzymes have been characterized from a variety of sources, which include humans (Carr et al., 1993; Hager et al., 1995; Hedstrom, 2009), protozoan parasites (Dobie et al., 2007) and bacteria (Ashbaugh \& Wessels, 1995; Park et al., 2004; Tiedeman \& Smith, 1985; Zhang et al., 2009). Many bacteria harbour a single gene 


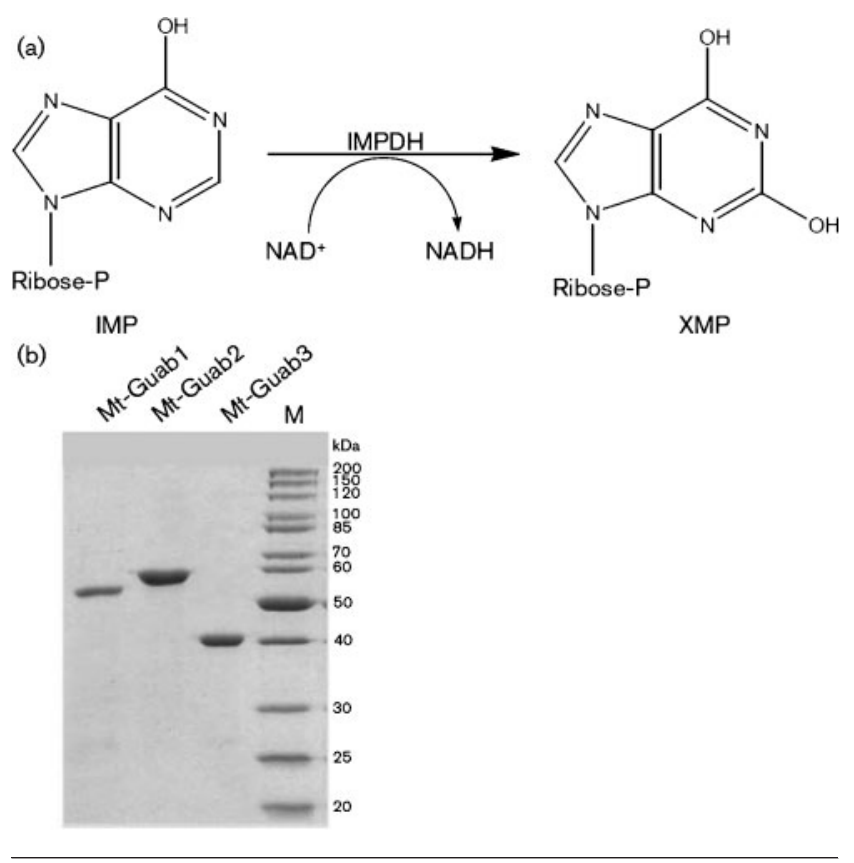

Fig. 1. (a) Reaction scheme of IMPDH. (b) Purification of recombinant $M$. tuberculosis GuaB orthologues expressed in $E$. coli. Purified Mt-GuaB1, Mt-GuaB2 and Mt-GuaB3 fractions from $\mathrm{Ni}^{2+}$-chelate affinity chromatography were pooled and analysed by $12 \%$ SDS-PAGE followed by Coomassie brilliant blue G-250 staining. M, Molecular mass markers.

for IMPDH, which is the sole provider of XMP for the cell and is thus vital for survival.

The Mycobacterium tuberculosis $\mathrm{H} 37 \mathrm{Rv}$ genome contains three genes predicted to encode IMPDH enzymes, designated guaB1 (Rv1843c), guaB2 (Rv3411c) and guaB3 (Rv3410c) (Cole et al., 1998). It is interesting to note that of the three orthologues of IMPDH, guaB3 and guaB2 are in an operon, and guaB1 is elsewhere in the genome. The Mt-GuaB orthologues have approximately $41 \%$ sequence identity at the amino acid level with human IMPDH (Shu \& Nair, 2008). A genome-wide transposon mutagenesis study has suggested that Mt-GuaB2 is the only essential orthologue for growth of M. tuberculosis H37Rv (Sassetti et al., 2001, 2003). In this study, we have biochemically characterized the MtGuaB orthologues, and have shown that Mt-GuaB2 is a drug target which is inhibited by a series of diphenyl urea-based compounds with potent M. tuberculosis activity.

\section{METHODS}

Materials. All restriction enzymes were purchased from New England Biolabs. Oligonucleotide primers were synthesized by MWG operon. All chemicals were obtained from Sigma. Diphenyl urea (DPU) analogues, 7834535 (DPU-1), 7285642 (DPU-2) and 7275166 (DPU-3) were purchased from ChemBridge.

Cloning of Mt-guaB orthologues in Escherichia coli and Mycobacterium smegmatis. The $M$. tuberculosis guaB genes were amplified by conventional PCR (see Supplementary Table S1 for
pET28b cloning primers and Supplementary Table S2 for pVV16 cloning primers, both available with the online version of this paper) and $M$. tuberculosis $\mathrm{H} 37 \mathrm{Rv}$ genomic DNA as a template. The purified PCR products of guaB2 and guaB3 were digested with NdeI and HindIII, and ligated into similarly digested expression vector $\mathrm{pET} 28 \mathrm{~b}$ (Novagen). The guaB1 PCR product was digested with EcoRI and HindIII and ligated into the pUC19 vector cut similarly and later subcloned into pET28b. The resulting recombinant plasmids were transformed into E. coli TOP-10 cells and subsequently recovered using QIAprep spin miniprep kit (Qiagen). For the pVV16 cloning of Mt-guaB orthologues, the PCR products were digested with NdeI and HindIII and cloned into pVV16 vector which had been digested with the same restriction enzymes, transformed into E. coli TOP-10 cells and plated on LB containing $25 \mu \mathrm{g}$ kanamycin $\mathrm{ml}^{-1}$ and $50 \mu \mathrm{g}$ hygromycin $\mathrm{ml}^{-1}$. The presence of an insert in positive clones was determined by digesting the plasmid with NdeI and HindIII or EcoRI and HindIII and separation on a $1 \%$ agarose gel. The correct nature of the insert was confirmed by sequencing.

Functional expression of $M$. tuberculosis IMPDH orthologues cloned in E. coli. Plasmids from positive clones were transformed into E. coli C41 (DE3) cells. A single colony was grown overnight at $37{ }^{\circ} \mathrm{C}$ using Luria-Bertani broth (LB; Difco) supplemented with $25 \mu \mathrm{g}$ kanamycin $\mathrm{ml}^{-1}$. A $1 \%$ inoculum of the overnight culture was inoculated into fresh LB medium containing $25 \mu \mathrm{g}$ kanamycin $\mathrm{ml}^{-1}$ and incubated at $37{ }^{\circ} \mathrm{C}$ until the culture reached $\mathrm{OD}_{600}$ 0.6-0.7. The culture was then cooled to $16{ }^{\circ} \mathrm{C}$ for $15 \mathrm{~min}$ before addition of $1 \mathrm{mM}$ IPTG and incubation was continued for a further $20 \mathrm{~h}$ at $16{ }^{\circ} \mathrm{C}$. The culture was harvested by centrifugation at $3500 \mathrm{~g}$ for $10 \mathrm{~min}$ at $4{ }^{\circ} \mathrm{C}$ and the resulting cell pellet was stored at $-80{ }^{\circ} \mathrm{C}$.

Purification of recombinant Mt-GuaB orthologues. The E. colicell pellets were resuspended in buffer A [50 mM Tris/ $\mathrm{HCl} \mathrm{pH} \mathrm{8.0,} 500 \mathrm{mM}$ $\mathrm{NaCl}, 10 \mathrm{mM}$ imidazole, $10 \%$ glycerol, $1 \mathrm{mM}$ dithiothreitol (DTT), $1 \mathrm{mM}$ phenylmethylsulfonyl fluoride (PMSF), $1 \mathrm{mM}$ benzamidine and lysozyme $\left(1 \mathrm{mg} \mathrm{ml}^{-1}\right)$ ] and stored for $30 \mathrm{~min}$ on ice. The cells were disrupted by sonication using eight pulses of $30 \mathrm{~s}$ with $30 \mathrm{~s}$ cooling intervals between pulses. The unbroken cells and cell debris were removed by centrifugation at $27000 \mathrm{~g}$ for $45 \mathrm{~min}$ at $4{ }^{\circ} \mathrm{C}$. The clear supernatant was applied to a $1 \mathrm{ml}$ chelating Sepharose HisTrap column charged with $\mathrm{Ni}^{2+}$ that had been equilibrated with buffer B (buffer A minus lysozyme and protease inhibitors). The column was extensively washed with buffer B before elution with 150 and $200 \mathrm{mM}$ imidazole in the same buffer. Fractions containing recombinant protein were pooled and dialysed against $50 \mathrm{mM}$ Tris/ $\mathrm{HCl} \mathrm{pH} 8.0,150 \mathrm{mM} \mathrm{NaCl}, 10 \%$ glycerol, $1 \mathrm{mM}$ EDTA and $1 \mathrm{mM}$ DTT and concentrated using a Centricon concentrator (Amicon). The concentrated enzyme was stored at $-80{ }^{\circ} \mathrm{C}$. Protein concentration was determined using the bicinchonic acid (BCA) protein assay kit (Pierce). The purified Mt-GuaB orthologues were analysed by $12 \%$ SDS-PAGE and stained with Coomassie brilliant blue R250.

MIC of DPU compounds against Mt-GuaB orthologues overexpressed in M. smegmatis and wild-type M. tuberculosis H37Rv. The susceptibility of wild-type M. smegmatis, M. smegmatis harbouring either the control pVV16 vector or pVV16-Mt-guaB1, pVV16-Mt-guaB2 and pVV16-Mt-guaB3 plasmids was determined in the presence of different concentrations of DPU-2 and DPU-3. The Mt-GuaB overexpressed and control strains were grown in tryptic soy broth (TSB) containing $0.05 \%$ Tween 80 at $37{ }^{\circ} \mathrm{C}$ until $\mathrm{OD}_{600}$ 0.7. Serial tenfold dilutions $\left(10^{-1}-10^{-8}\right)$ of each culture were spotted $(5 \mu \mathrm{l})$ on TSB agar plates supplemented with $25 \mu \mathrm{g}$ kanamycin $\mathrm{ml}^{-1}$ and $50 \mu \mathrm{g}$ hygromycin $\mathrm{ml}^{-1}$ and serial twofold dilutions of the respective DPU compound ranging from 0.125 to $8 \mu \mathrm{g} \mathrm{ml}^{-1}$. Plates were incubated at $37^{\circ} \mathrm{C}$ for $3-$ 4 days. The MIC was defined as the minimal concentration that inhibited $99 \%$ of bacilli growth. Lysates of pVV16 vector control and Mt-GuaB overexpressed strains were prepared and the expression was confirmed by Western blotting and immunological detection with anti- 
His tag antibodies (Qiagen) according to the manufacturer's protocol. The MIC of DPU compounds for wild-type M. tuberculosis H37Rv was determined essentially as described by measurement of AlamarBlue fluorescence (Ananthan et al., 2009; Maddry et al., 2009). The DPU compounds were screened initially at $10 \mu \mathrm{g} \mathrm{ml}^{-1}$ and later at a doseresponse with serial twofold dilutions covering a range from 0.1 to $50 \mu \mathrm{g}$ $\mathrm{ml}^{-1}$ (Ananthan et al., 2009; Maddry et al., 2009).

Enzyme assays. IMPDH activity was assayed at $37^{\circ} \mathrm{C}$ in a heatcontrolled UV spectrophotometer (Jenway) by following the production of NADH $\left(\varepsilon_{340}=6.22 \mathrm{mM}^{-1} \mathrm{~cm}^{-1}\right)$ at $340 \mathrm{~nm}$ in a $1 \mathrm{~cm}$ path length cuvette. After pre-incubation of the reaction mixture for $10 \mathrm{~min}$ at $37^{\circ} \mathrm{C}$ to achieve thermal equilibration, the reaction was started by the addition of purified enzyme and incubated at $37^{\circ} \mathrm{C}$ for $20 \mathrm{~min}$. The IMPDH activity was assayed essentially as described by $\mathrm{Pal}$ et al. (2002) with minor modifications. The $1 \mathrm{ml}$ reaction mixture consisted of $50 \mathrm{mM}$ Tris/HCl pH 8.5, $150 \mathrm{mM} \mathrm{KCl,} 1.25 \mathrm{mM} \mathrm{IMP,} 3 \mathrm{mM} \mathrm{NAD}^{+}, 1 \mathrm{mM}$ DTT, $1 \mathrm{mM}$ EDTA and a final enzyme concentration of $4 \mu \mathrm{g}$. Control experiments contained all the assay components except the enzyme or substrate (minus IMP or $\mathrm{NAD}^{+}$). Assays were performed in duplicate. One unit of enzyme activity was defined as the amount of enzyme required to transform $1 \mu$ mole substrate $\min ^{-1}$ at $37^{\circ} \mathrm{C}$. The specific activity was expressed as $\mu$ mole $\mathrm{NADH}$ produced $\mathrm{min}^{-1}$ (mg protein $)^{-1}$.

$K_{\mathrm{m}}$ (app) determination experiments for $\mathrm{Mt}-\mathrm{GuaB} 2$ in the forward reaction were carried out by varying the concentration of one substrate while the concentration of the second substrate was maintained at a constant saturating level. The apparent $K_{\mathrm{m}}$ values for IMP and $\mathrm{NAD}^{+}$ were assessed from the initial rate measurements in duplicate by nonlinear curve fitting using the Graphpad prism software. For MtGuaB1, Mt-GuaB2 and Mt-GuaB3, the concentration of IMP was varied between 0.01 and $3 \mathrm{mM}, \mathrm{NAD}^{+}$was varied between 0.03 and $4.5 \mathrm{mM}$ with a final enzyme concentration of $4 \mu \mathrm{g}$. The $\mathrm{pH}$ optimum for the purified recombinant Mt-GuaB2 was determined by performing the assay at various $\mathrm{pH}$ values with increments of $0.5 \mathrm{pH}$ units. The thermal stability of the protein was determined by pre-incubating Mt-GuaB2 at a variety of temperatures for $10 \mathrm{~min}$ and then the reaction mixture was added to start the assay. To determine the effect of temperature on enzyme activity, the reactions were incubated at different temperatures for $20 \mathrm{~min}$ at $\mathrm{pH}$ 8.5. The effect of monovalent cations $(\mathrm{KCl}, \mathrm{RbCl}$, $\mathrm{CsCl}$ and $\mathrm{NH}_{4} \mathrm{Cl}$ ) on Mt-GuaB2 activity was also examined at a concentration of $150 \mathrm{mM}$. The enzymic activity of Mt-GuaB2 was measured in the presence of three DPU compounds (DPU-1, DPU-2 and DPU-3). The inhibition constant, $K_{\mathrm{i}}$, of the DPU inhibitors were measured at saturating amounts of $\mathrm{NAD}^{+}\left(3 \mathrm{mM} ; K_{\mathrm{m}} 610.5 \mu \mathrm{M}\right)$, with varying concentrations of $\operatorname{IMP}\left(64,128\right.$ and $\left.256 \mu \mathrm{M} ; K_{\mathrm{m}} 128.1 \mu \mathrm{M}\right)$ in the absence and presence of increasing concentrations of DPU-1 $(0,100$, $300,500,650$ and $800 \mu \mathrm{M}), \mathrm{DPU}-2(0,300,1000,1300,1600$ and $2000 \mu \mathrm{M})$ or DPU-3 $(0,10,30,100,300$ and $1000 \mu \mathrm{M})$. The reactions were initiated by the addition of $\mathrm{NAD}^{+}$and the absorbance was measured at $340 \mathrm{~nm}$ using the assay as described above. The initial velocity $(\mathrm{V})$ without inhibitor and at each inhibitor concentration was plotted on the $y$-axis versus IMP concentration on the $x$-axis. $K_{\mathrm{i}}$ (for noncompetitive inhibition) and alpha $K_{\mathrm{i}}$ (for uncompetitive inhibition) values were determined from non-linear regression analysis using the Graph pad prism program by fitting data to the equation for noncompetitive and uncompetitive inhibition, respectively.

\section{RESULTS}

\section{Expression and purification of recombinant Mt- GuaB orthologues cloned in E. coli}

To compare the three GuaB orthologues in M. tuberculosis $\mathrm{H} 37 \mathrm{Rv}$, the genes encoding these proteins were cloned and expressed in the heterologous host E. coli as hexa-histidine fusion proteins using the pET28b vector under the control of a T7 promoter. E. coli C41 (DE3) cells were transformed with the pET28b-guaB constructs. To prevent inclusion body formation, it was necessary to induce expression of recombinant protein production at $16{ }^{\circ} \mathrm{C}$ for $20 \mathrm{~h}$. Under these conditions, sufficient soluble protein was produced and subsequently purified using HisTrap $\mathrm{Ni}^{2+}$ chelating Sepharose affinity chromatography (Fig. 1b). The Mt$\mathrm{GuaB}$ orthologues were purified with a satisfactory yield of approximately $1.7 \mathrm{mg}$ (Mt-GuaB1), $3.3 \mathrm{mg}$ (Mt-GuaB2) and $1.8 \mathrm{mg}$ (Mt-GuaB3) per litre of culture. To confirm that the cloned genes were indeed IMPDHs, the activities of Mt-GuaB1, Mt-GuaB2 and Mt-GuaB3 were assayed spectrophotometrically.

\section{Biochemical properties of recombinant Mt-GuaB orthologues}

Analysis of the purified recombinant Mt-GuaB1, Mt-GuaB2 and Mt-GuaB3 revealed that only Mt-GuaB2 (Rv3411c) encoded a functional IMPDH (see below). The purified enzymes from the other two orthologues, Mt-GuaB1 and Mt-GuaB3, lacked IMPDH activity (data not shown).

The Mt-GuaB2 enzyme exclusively catalysed the oxidative deamination (forward reaction) of IMP in the presence of $\mathrm{NAD}^{+}$. In the absence of enzyme or substrates (IMP or $\mathrm{NAD}^{+}$) no activity was detectable. The optimum protein concentration for Mt-GuaB2 activity was determined to be $4 \mu \mathrm{g} \mathrm{ml}^{-1}$ which was in the most linear part of the curve (Fig. 2a). The optimal temperature for catalytic activity was $37^{\circ} \mathrm{C}$ (Fig. 2b). To determine the $\mathrm{pH}$ activity profile of Mt-GuaB2, the enzyme activity was monitored at saturating substrate conditions at several different $\mathrm{pH}$ values (Fig. 2c). The $\mathrm{pH}$ optima of Mt-GuaB2 activity was determined to be approximately 8-8.5 (Fig. 2c). Mt-GuaB2 enzyme could be stored at $-80{ }^{\circ} \mathrm{C}$ and $\mathrm{pH} 8.5$ for several months with negligible loss of enzymic activity. The enzyme was thermostable and retained activity upon incubation at $60{ }^{\circ} \mathrm{C}$ for $10 \mathrm{~min}$ (Fig. 2d). Above $70{ }^{\circ} \mathrm{C}$, there was a complete inhibition in activity due to thermal inactivation of the enzyme (Fig. 2d).

The presence of DTT and EDTA, typically used in the routine assays, had no stimulatory effect on Mt-GuaB2 activity. Monovalent cations, such as $\mathrm{K}^{+}$, activate IMPDH (Hedstrom, 2009; Kerr et al., 2000; Zhou et al., 1997) and in a similar fashion Mt-GuaB2 exhibited a requirement for $\mathrm{KCl}$ (Fig. 3a). In order to investigate the effect of other salts on Mt-GuaB2 activity, the reaction was carried out in the presence of $\mathrm{NH}_{4} \mathrm{Cl}, \mathrm{RbCl}$ and $\mathrm{CsCl}$. The presence of $\mathrm{RbCl}$ or $\mathrm{NH}_{4} \mathrm{Cl}$ at the same concentration was also stimulatory but to a lesser extent than that observed for $\mathrm{KCl}$ (Fig. 3b).

\section{Determination of apparent kinetic parameters of Mt-GuaB2}

The apparent kinetic parameters for IMPDH activity of Mt-GuaB2 were determined at the optimum $\mathrm{pH}$ of 8.5. 

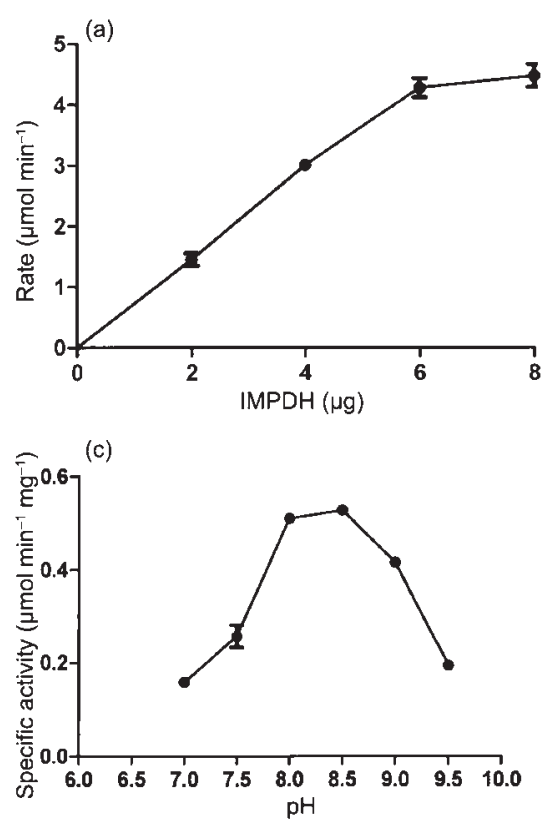

(b)
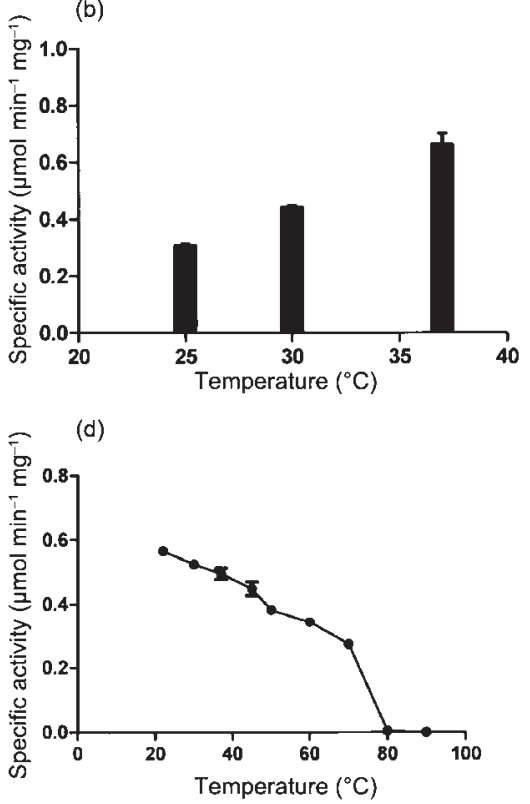

Fig. 2. Characterization of recombinant MtGuaB2 expressed in E. coli. (a) Protein doseresponse. (b) Optimum assay temperature. (c) $\mathrm{pH}$ optimum. (d) Thermotolerance of MtGuaB2. After pre-incubation of the reaction mixture for $10 \mathrm{~min}$ at $37{ }^{\circ} \mathrm{C}$ the reaction was started by the addition of purified enzyme and incubated at $37{ }^{\circ} \mathrm{C}$ for $20 \mathrm{~min}$. (d) Thermotolerance of Mt-Guab2. Recombinant Mt-Guab2 $(4 \mu \mathrm{g})$ was pre-incubated at various temperatures for $10 \mathrm{~min}$ in the reaction buffer before starting the reaction by adding the substrate. The remaining activity was assayed at $37{ }^{\circ} \mathrm{C}$. Data shown are mean \pm SEM.
Plots of initial velocity versus either IMP or $\mathrm{NAD}^{+}$ concentration showed Michaelis-Menten kinetics. The apparent Michaelis constant $\left(K_{\mathrm{m}}\right)$ values for the substrates IMP and $\mathrm{NAD}^{+}$were calculated to be $128.1 \pm 10.85 \mu \mathrm{M}$ and $610.5 \pm 69.16 \mu \mathrm{M}$, respectively. The $K_{\mathrm{m}}$ value for IMP was close to that observed with Streptococcus pyogenes IMPDH which had a $K_{\mathrm{m}}$ of $62 \mu \mathrm{M}$ (Prosise et al., 2002; Zhang et al., 1999). Generally, bacterial IMPDHs have been reported to have higher $K_{\mathrm{m}}$ values for IMP and $\mathrm{NAD}^{+}$ than non-bacterial enzymes (Kerr \& Hedstrom, 1997; Umejiego et al., 2004; Zhou et al., 1997).

The results established that Mt-GuaB2 encodes a functional enzyme that possesses IMPDH activity. The enzyme did not catalyse the reverse reaction, i.e. the conversion of XMP to IMP. Product inhibition of the forward reaction by XMP and NADH is characteristic of several IMPDH enzymes studied to date (Verham et al., 1987; Xiang et al., 1996). The Mt-GuaB2 enzyme exhibited substrate inhibition in vitro at high concentrations of NADH and XMP. The activity was strongly inhibitory with more than $95 \%$ inhibition at a concentration of $1 \mathrm{mM} \mathrm{NADH}$. Higher concentrations of NADH completely abolished Mt-GuaB2 activity. The in vitro activity was also $40 \%$ inhibited by $\mathrm{XMP}$ at a concentration of $1 \mathrm{mM} \mathrm{XMP.}$

\section{Inhibition studies}

Screening results for over 100000 compounds from the ChemBridge collection of drug-like molecules has been made publicly accessible through PubChem (http:// pubchem.ncbi.nlm.nih.gov/) and has been reported recently (Ananthan et al., 2009). A cursory informatic analysis of the numerous hits was done which identified a number of potentially interesting compounds and their targets (Ananthan et al., 2009). Among these, the DPU compounds are known inhibitors of kinases and IMPDH (Ananthan et al., 2009). Ideally, the known, potent activity against $M$. tuberculosis in vitro would be an ideal starting point for drug design presuming a target can be identified. Typically, antibacterial drug design programs have been hindered when starting from enzyme-based inhibition and later building on antibacterial activity. Unfortunately, pure phenotypic screens often lead to inhibitors that require
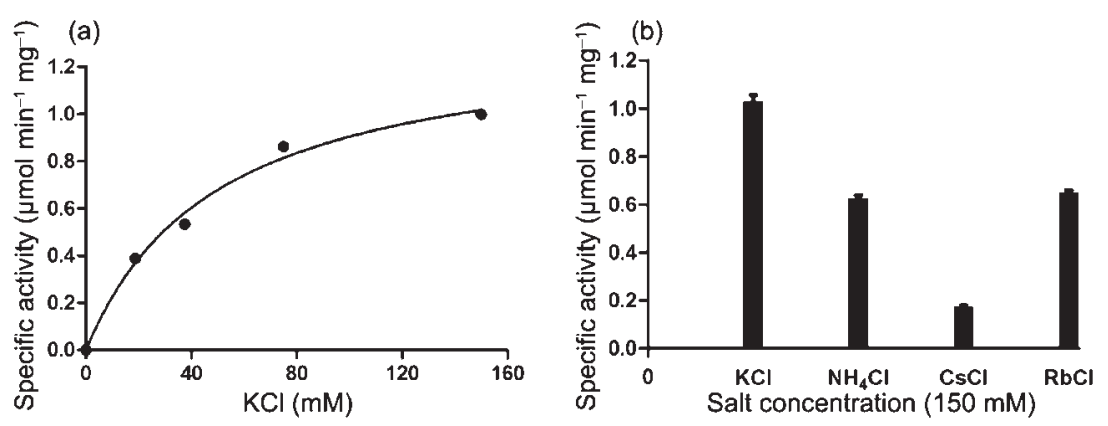

Fig. 3. $\mathrm{K}^{+}$dependence (a) and monovalent cation dependence (b) of recombinant MtGuaB2 activity. The initial velocities of IMPDH activity were measured by varying the concentration of $\mathrm{KCl}$ between 18.75 and $150 \mathrm{mM}$. The activities of each monovalent cation were determined at a fixed concentration of $150 \mathrm{mM}$. The specific activity was expressed as $\mu \mathrm{mol}$ $\mathrm{NADH}$ produced $\mathrm{min}^{-1}$ (mg protein) ${ }^{-1}$. 
extensive investigation prior to identification of an optimal and specific enzyme activity.

With the hypothesis in mind that these types of molecules (DPUs) inhibited IMPDH, we purchased a small set of the more active and selective samples that were available from ChemBridge. A total of 16 DPU analogues were initially screened at a concentration of $1 \mathrm{mM}$ in the in vitro MtGuaB2 activity assay. Among the analogues screened, three compounds (DPU-1, DPU-2 and DPU-3) displayed $>90 \%$ inhibition at $1 \mathrm{mM}$. These three compounds were retested at different concentrations in order to determine $K_{\mathrm{i}}$ and alpha $K_{\mathrm{i}}$ values for each compound (Table 1 ). The alpha $K_{\mathrm{i}}$ values of DPU-1 and DPU-3 were $231.8 \mu \mathrm{M}$ and $181.2 \mu \mathrm{M}$, respectively, and the $K_{\mathrm{i}}$ value of DPU-2 was $1203 \mu \mathrm{M}$. DPU1 and -3 showed uncompetitive inhibition with respect to IMP, and DPU-2 showed noncompetitive inhibition versus IMP (Fig. 4a-c). The Lineweaver-Burk plots (Fig. 4a-c, inset) were generated only to indicate the type of inhibition observed. The three inhibitors were also tested for their ability to inhibit whole cell growth of $M$. tuberculosis and $M$. smegmatis. All three inhibitors were found to possess antimycobacterial activity against $M$. tuberculosis $\mathrm{H} 37 \mathrm{Rv}$ (Table 1) with MIC values ranging from 0.2 to $0.4 \mu \mathrm{g} \mathrm{ml}^{-1}$. DPU-2 and -3 inhibited M. smegmatis growth to a similar extent, with an MIC value of $0.5 \mu \mathrm{g} \mathrm{ml}^{-1}$ for each compound. DPU1 did not inhibit $M$. smegmatis growth even at the highest concentration $\left(40 \mu \mathrm{g} \mathrm{ml}^{-1}\right)$ tested.

\section{Drug susceptibility of Mt-GuaB orthologues overexpressed in M. smegmatis}

To confirm whether Mt-GuaB and IMPDH activity is the target of the DPU compounds (Table 1), the M. smegmatis pVV16 overexpressing strains were screened for susceptibility to the DPU compounds (Table 2 and Fig. 5a). The overexpressed Mt-GuaB orthologues in $M$. smegmatis showed increased resistance to DPU-2 and -3 compared with the pVV16 vector control (Table 2 and Fig. 5a). The MIC values of DPU-3 against pVV16-Mt-guaB1, -MtguaB2 and -Mt-guaB3 strains were 16-fold higher than that of the pVV16 vector control (Table 2). The data indicated that the target protein of the DPU compounds was indeed IMPDH. The MIC of M. smegmatis to compound DPU-2 was enhanced twofold, indicating that the overproduction of Mt-GuaB orthologues also conferred resistance to compound DPU-2 (Table 2). It is interesting that although Mt-GuaB1 and Mt-GuaB3 were functionally inactive, the above gene dosage experiment suggests that the DPU compounds presumably bind to Mt-GuaB1 and Mt-GuaB3 proteins, thus requiring a higher concentration for inhibition. The Western blot after immunological detection with anti-His tag antibody clearly confirmed the expression in the pVV16-Mt-GuaB1-3 constructs (Fig. 5b).

\section{DISCUSSION}

Purine biosynthetic enzymes, and IMPDH in particular, are considered to be attractive targets for development of novel anti-mycobacterial agents. To further our knowledge of purine metabolism we have investigated the IMPDH enzyme of $M$. tuberculosis. IMPDH, encoded by the guaB gene catalyses the reversible conversion of IMP to XMP. In E. coli, at least two different enzymic pathways lead to the synthesis of XMP (Neuhard \& Nygaard, 1996; Pimkin et al., 2009) which are well characterized (Gilbert et al., 1979; Gilbert \& Drabble, 1980; Kerr \& Hedstrom, 1997; Kerr et al., 2000; Tiedeman \& Smith, 1985). However, the M. tuberculosis $\mathrm{H} 37 \mathrm{Rv}$ purine de novo biosynthesis pathway enzymes have received little attention to date and are poorly studied.

Each of the three Mt-guaB genes was successfully expressed in E. coli and M. smegmatis. In both E. coli and $M$.

Table 1. Structures of DPU analogues and their corresponding MIC values against M. tuberculosis and M. smegmatis, and the $K_{\mathrm{i}}$ and alpha $K_{\mathrm{i}}$ values obtained for the in vitro inhibition of Mt-GuaB2 purified from E. coli

Compound




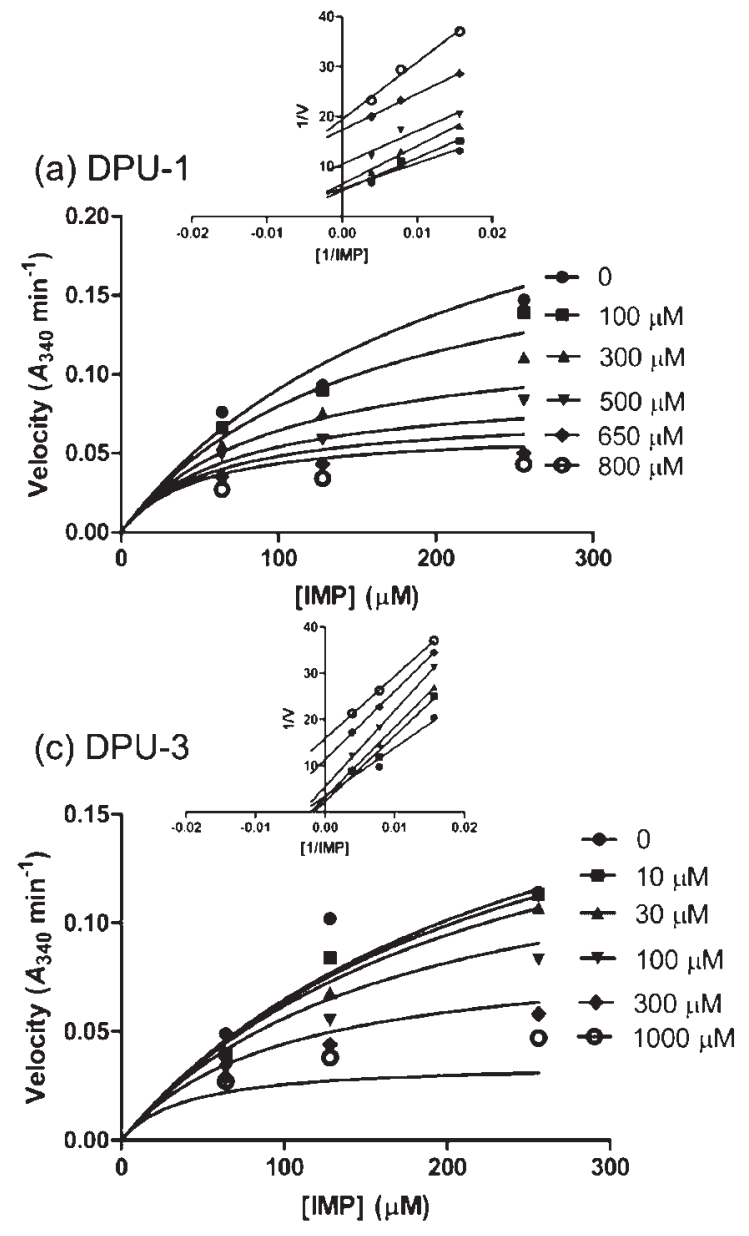

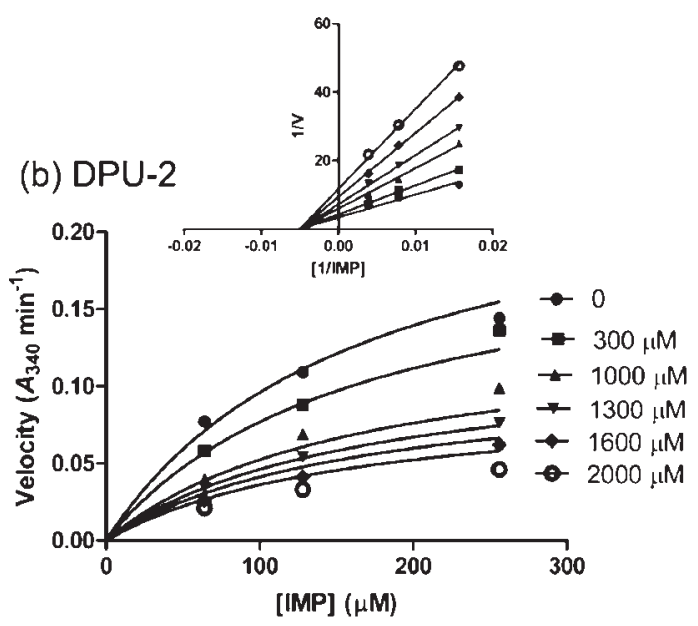

Fig. 4. The mechanism of inhibition of DPU-1 (a), DPU-2 (b) and DPU-3 (c) by IMP $(64-256 \mu \mathrm{M})$. Mt-GuaB2 was assayed as described in Methods in the absence of inhibitor and with increasing concentrations of DPU-1, -2 and -3 . Data were fitted in Graphpad prism to a non-linear regression function for uncompetitive (DPU-1 and -3) and noncompetitive (DPU-2) inhibition. The Lineweaver-Burk plots inset (top) of each plot were generated only to display the type of inhibition. smegmatis, only the recombinant overexpressed Mt-GuaB2 exhibited IMPDH activity. Mt-GuaB1 and Mt-GuaB3 were obtained as soluble proteins but they lacked enzymic activity. The biochemical properties of the functional orthologue Mt-GuaB2 were extensively characterized.

Table 2. MIC of DPU-2 and -3 against Mt-GuaB pVV16overexpressed $M$. smegmatis strains

\begin{tabular}{|lcc|}
\hline \multirow{2}{*}{ M. smegmatis strain } & \multicolumn{2}{c|}{ MIC $_{\mathbf{9 0}}{ }^{*}\left(\boldsymbol{\mu \mathbf { g ~ m l } ^ { - \mathbf { 1 } } )}\right.$} \\
\cline { 2 - 3 } & DPU-3 & DPU-2 \\
\hline mc $^{2} 155$ & 0.5 & 0.5 \\
pVV16 & 0.5 & 0.5 \\
pVV16:Mt-guaB1 & 8 & 1 \\
pVV16:Mt-guaB2 & 8 & 1 \\
pVV16:Mt-guaB3 & 8 & 1 \\
\hline
\end{tabular}

${ }^{*}$ The $\mathrm{MIC}_{90}$ value is expressed as the lowest concentration that inhibited visible growth of the organism on a TSB agar plate after incubation at $37{ }^{\circ} \mathrm{C}$ for 3-4 days.
Mt-GuaB2 had an absolute substrate specificity for IMP and $\mathrm{NAD}^{+}$. The $K_{\mathrm{m}}$ values calculated for $\mathrm{NAD}^{+}$and IMP were within the range of $K_{\mathrm{m}}$ values determined for other known bacterial IMPDH enzymes (Kerr \& Hedstrom, 1997; Prosise et al., 2002; Zhang et al., 1999). The specific features of the Mt-GuaB2 enzyme were its elevated stability towards higher temperatures, higher $\mathrm{pH}$ optimum and requirement of $\mathrm{K}^{+}$for functional activity.

Our results led us to further investigate and attempt to explain the apparent non-functional nature of Mt-GuaB1 and Mt-GuaB3. Bioinformatic analysis of the three homologue sequences (and their comparison with that of human type II IMPDH) reveals potential reasons why Mt-GuaB2 is the only active orthologue amongst the three M. tuberculosis orthologues. The complex of human type II IMPDH with 6-chloropurine riboside 5'-monophosphate and $\mathrm{NAD}^{+}$(PDB ID, 1NFB; Risal et al., 2004) identifies several important active site residues, which can be mapped to Mt-GuaB1-3 in a multiple sequence alignment (Fig. 6). Most significantly, the Cys nucleophile that forms a covalent intermediate with the substrate is altered to Thr in Mt-GuaB3 (Thr226), and so would presumably be unable to initiate the first stage of catalysis. Furthermore, 
(a) DPU-3 $\left(8 \mu \mathrm{g} \mathrm{ml}^{-1}\right)$
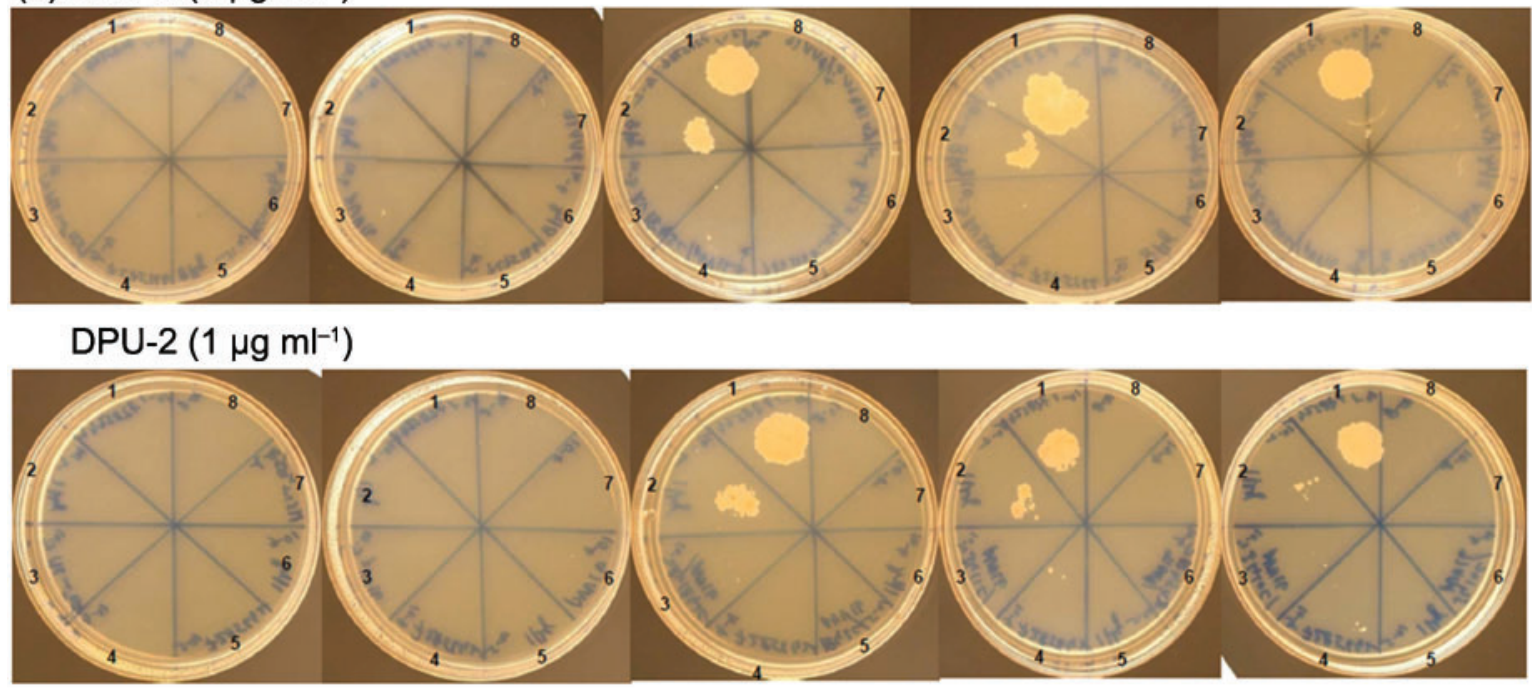

M. smegmatis $\mathrm{mc}^{2} 155$

M. smegmatis pV16

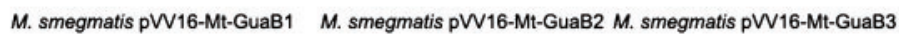

(b)

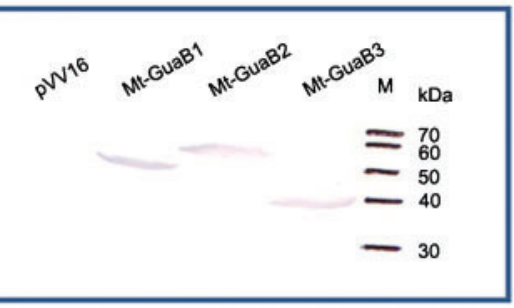

Fig. 5. Growth of Mt-GuaB1, Mt-GuaB2 and Mt-GuaB3 overexpressing strains in the presence of $8 \mu \mathrm{g}$ DPU-3 $\mathrm{ml}^{-1}$ and $1 \mu \mathrm{g}$ DPU-2 $\mathrm{ml}^{-1}$. (a) pVV16 vector and the Mt-GuaB1-3 overexpression strains were transformed into M. smegmatis and grown in TSB containing $0.05 \%$ Tween 80 , until $\mathrm{OD}_{600}$ 0.7. Numbers $1-8$ indicate serial tenfold dilutions $\left(10^{-1}-10^{-8}\right)$ of each culture which were spotted on TSB plates supplemented with kanamycin, hygromycin and the DPU compound. (b) Western blot analysis of lysates of Mt-GuaB overexpressing M. smegmatis strains and pVV16 vector, to confirm expression.

Mt-GuaB3 lacks two conserved residues that contact the nicotinamide headgroup of $\mathrm{NAD}^{+}$; human IMPDH residues Asp274 and Arg322 have Asp and Lys counterparts in Mt-GuaB1 and Mt-GuaB2, but align with Gln164 and Ile216 in Mt-GuaB3. A structural basis for the non-observable activity of Mt-GuaB1 is less obvious, but may come from a shift that alters the position of a conserved Tyr; human IMPDH Tyr411 contacts the edge of the nucleotide substrate and sits alongside the Cys nucleophile, but aligns with Ser384 in Mt-GuaB1. An experimental structure of Mt-GuaB1 would be required to confirm this, in particular determining that the neighbouring Tyr385 does not occupy this position. Another possibility for the lack of IMPDH activity in Mt-GuaB1 and Mt-GuaB3 may relate to the hydrolytic half-reaction, as only Mt-GuaB2 possesses an equivalent residue to Arg412 of human type II IMPDH postulated to activate a water molecule implicated in the hydrolysis of the covalent enzyme intermediate (Hedstrom, 2009). The metabolic function of Mt-GuaB1 and Mt-GuaB3 awaits further clarification; however, several 'moonlighting' roles for
IMPDH enzymes independent of classical catalytic activity have been proposed (Hedstrom, 2009).

We have shown that the DPU analogues exhibited potent anti-mycobacterial activity against $M$. tuberculosis and $M$. smegmatis growth. The mode of action of the DPU compounds was through in vitro and in vivo inhibition of Mt-GuaB2, and we established that Mt-GuaB orthologues were indeed the targets for the DPUs examined. However, the presence of other potential cellular targets may exist. Surprisingly, DPU-1, which was an effective Mt-GuaB2 inhibitor, did not inhibit M. smegmatis growth even at very high concentrations, although it inhibited $M$. tuberculosis. Antimycobacterial drugs, such as isoniazid and rifampicin have also been shown to have higher MIC values against M. smegmatis compared with $M$. tuberculosis (Chaturvedi et al., 2007; Chung et al., 1995; Reddy et al., 2008). One possible explanation for this may be cell wall permeability, which is the major barrier and factor contributing to the intrinsic resistance of M. smegmatis to rifampicin (Hetherington et al., 1995; Jarlier \& Nikaido, 

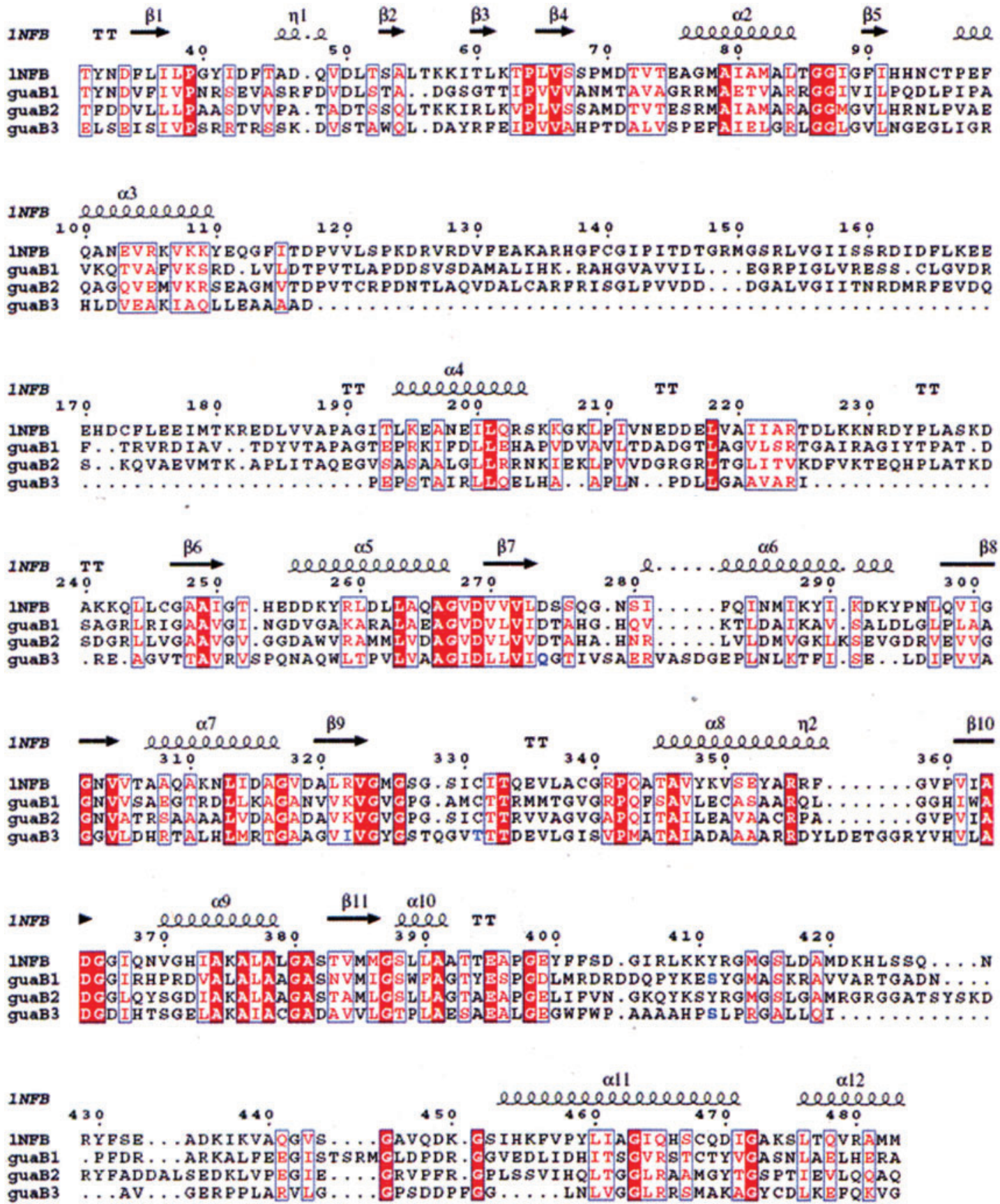

Fig. 6. Comparative sequence alignment of Mt-Guab1-3 and the human type II IMPDH. Possible amino acid differences in MtGuaB1 and Mt-GuaB3 responsible for a lack of IMPDH activity referred to in the text are blue. Partially conserved residues are in red, conserved residues are white on a red background. Numbering and secondary structure used above the alignment are taken from human type II IMPDH (PDB 1NFB).

1994; Piddock et al., 2000). Resistance of M. smegmatis to isoniazid and rifampicin has also been attributed to drug efflux mechanisms (Chaturvedi et al., 2007; Li et al., 2004; Piddock et al., 2000). Remarkably, these DPU inhibitors of Mt-GuaB2 have mechanistic and structural precedent in several compounds identified through high-throughput screening of Cryptosporidium parvum IMPDH (Macpherson et al., 2010). Despite the protozoan nature of C. parvum, its IMPDH has long been thought to have been acquired via gene transfer from a bacterial source (Striepen et al., 2002; Umejiego et al., 2004). Observing the structure of $C$. parvum IMPDH with one of these inhibitors (inhibitor C64, PDB code $3 \mathrm{KHJ}$ ) reveals a novel interaction with the enzyme, which partially stacks against the purine ring of IMP. A direct 
comparison of DPU-2 with C64 yields a good match for structurally equivalent chemical groups; the chloroaniline, urea-linker and nitrotoluene groups of DPU-2 are presumably analogous to the bromoaniline, amide linker and thiazole groups of C64, respectively. This significant agreement in inhibitor structure further validates our conclusion that MtGuaB2 is the target of these compounds, and may also provide a potential basis for the optimization of DPU1-3. The mechanism of inhibition of DPU-3 was uncompetitive versus IMP and that of DPU-2 was noncompetitive with respect to IMP. In summary, we conclude that DPU-2 and DPU-3 exhibit anti-mycobacterial activity by specifically inhibiting Mt-GuaB2.

\section{ACKNOWLEDGEMENTS}

G.S.B. acknowledges support in the form of a Personal Research Chair from Mr James Bardrick and a Royal Society Wolfson Research Merit Award, as a former Lister Institute-Jenner Fellow, and from the Medical Research Council. The pVV16 vector was a kind gift from Dr Varalakshmi Vissa, Colorado State University, Fort Collins, USA. Thanks to Mr Albel Singh of the University of Birmingham for taking the photographs of plates.

\section{REFERENCES}

Ananthan, S., Faaleolea, E. R., Goldman, R. C., Hobrath, J. V., Kwong, C. D., Laughon, B. E., Maddry, J. A., Mehta, A., Rasmussen, L. \& other authors (2009). High-throughput screening for inhibitors of Mycobacterium tuberculosis H37Rv. Tuberculosis (Edinb) 89, 334353.

Ashbaugh, C. D. \& Wessels, M. R. (1995). Cloning, sequence analysis and expression of the group A streptococcal guaB gene encoding inosine monophosphate dehydrogenase. Gene 165, 57-60.

Carr, S. F., Papp, E., Wu, J. C. \& Natsumeda, Y. (1993). Characterization of human type I and type II IMP dehydrogenases. J Biol Chem 268, 27286-27290.

Chaturvedi, V., Dwivedi, N., Tripathi, R. P. \& Sinha, S. (2007). Evaluation of Mycobacterium smegmatis as a possible surrogate screen for selecting molecules active against multi-drug resistant Mycobacterium tuberculosis. J Gen Appl Microbiol 53, 333-337.

Chung, G. A., Aktar, Z., Jackson, S. \& Duncan, K. (1995). Highthroughput screen for detecting antimycobacterial agents. Antimicrob Agents Chemother 39, 2235-2238.

Cole, S. T., Brosch, R., Parkhill, J., Garnier, T., Churcher, C., Harris, D., Gordon, S. V., Eiglmeier, K., Gas, S. \& other authors (1998). Deciphering the biology of Mycobacterium tuberculosis from the complete genome sequence. Nature 393, 537-544.

Corbett, E. L., Watt, C. J., Walker, N., Maher, D., Williams, B. G., Raviglione, M. C. \& Dye, C. (2003). The growing burden of tuberculosis: global trends and interactions with the HIV epidemic. Arch Intern Med 163, 1009-1021.

Dobie, F., Berg, A., Boitz, J. M. \& Jardim, A. (2007). Kinetic characterization of inosine monophosphate dehydrogenase of Leishmania donovani. Mol Biochem Parasitol 152, 11-21.

Gilbert, H. J. \& Drabble, W. T. (1980). Active-site modification of native and mutant forms of inosine 5 '-monophosphate dehydrogenase from Escherichia coli K12. Biochem J 191, 533-541.

Gilbert, H. J., Lowe, C. R. \& Drabble, W. T. (1979). Inosine 5'monophosphate dehydrogenase of Escherichia coli. Purification by affinity chromatography, subunit structure and inhibition by guanosine 5'-monophosphate. Biochem J 183, 481-494.

Hager, P. W., Collart, F. R., Huberman, E. \& Mitchell, B. S. (1995). Recombinant human inosine monophosphate dehydrogenase type I and type II proteins. Purification and characterization of inhibitor binding. Biochem Pharmacol 49, 1323-1329.

Hedstrom, L. (2009). IMP dehydrogenase: structure, mechanism, and inhibition. Chem Rev 109, 2903-2928.

Hetherington, S. V., Watson, A. S. \& Patrick, C. C. (1995). Sequence and analysis of the rpoB gene of Mycobacterium smegmatis. Antimicrob Agents Chemother 39, 2164-2166.

Jackson, R. C., Weber, G. \& Morris, H. P. (1975). IMP dehydrogenase, an enzyme linked with proliferation and malignancy. Nature 256, 331-333.

Jarlier, V. \& Nikaido, H. (1994). Mycobacterial cell wall: structure and role in natural resistance to antibiotics. FEMS Microbiol Lett 123, 11-18.

Kaufmann, S. H. \& Parida, S. K. (2007). Changing funding patterns in tuberculosis. Nat Med 13, 299-303.

Kerr, K. M. \& Hedstrom, L. (1997). The roles of conserved carboxylate residues in IMP dehydrogenase and identification of a transition state analog. Biochemistry 36, 13365-13373.

Kerr, K. M., Cahoon, M., Bosco, D. A. \& Hedstrom, L. (2000). Monovalent cation activation in Escherichia coli inosine 5' -monophosphate dehydrogenase. Arch Biochem Biophys 375, 131-137.

Lambden, P. R. \& Drabble, W. T. (1973). The gua operon of Escherichia coli K-12: evidence for polarity from guaB to guaA. J Bacteriol 115, 992-1002.

Li, X. Z., Zhang, L. \& Nikaido, H. (2004). Efflux pump-mediated intrinsic drug resistance in Mycobacterium smegmatis. Antimicrob Agents Chemother 48, 2415-2423.

Macpherson, I. S., Kirubakaran, S., Gorla, S. K., Riera, T. V., D’Aquino, J. A., Zhang, M., Cuny, G. D. \& Hedstrom, L. (2010). The structural basis of Cryptosporidium-specific IMP dehydrogenase inhibitor selectivity. J Am Chem Soc 132, 1230-1231.

Maddry, J. A., Ananthan, S., Goldman, R. C., Hobrath, J. V., Kwong, C. D., Maddox, C., Rasmussen, L., Reynolds, R. C., Secrist, J. A., III \& other authors (2009). Antituberculosis activity of the molecular libraries screening center network library. Tuberculosis (Edinb) 89, 354-363.

Magasanik, B., Moyed, H. S. \& Gehring, L. B. (1957). Enzymes essential for the biosynthesis of nucleic acid guanine; inosine $5^{\prime}$ phosphate dehydrogenase of Aerobacter aerogenes. J Biol Chem 226, 339-350.

Neuhard, J. \& Nygaard, P. (1996). Purines and Pyrimidines. In Escherichia coli and Salmonella typhimurium: Cellular and Molecular Biology, pp. 445-461. Edited by F. C. Neidhardt. Washington, DC: American Society for Microbiology.

Pal, S., Bera, B. \& Nair, V. (2002). Inhibition of inosine monophosphate dehydrogenase (IMPDH) by the antiviral compound, 2vinylinosine monophosphate. Bioorg Med Chem 10, 3615-3618.

Park, S. J., Joo, W. A., Choi, J., Lee, S. H. \& Kim, C. W. (2004). Identification and characterization of inosine monophosphate dehydrogenase from Halobacterium salinarum. Proteomics 4, 3632-3641.

Piddock, L. J., Williams, K. J. \& Ricci, V. (2000). Accumulation of rifampicin by Mycobacterium aurum, Mycobacterium smegmatis and Mycobacterium tuberculosis. J Antimicrob Chemother 45, 159-165.

Pimkin, M., Pimkina, J. \& Markham, G. D. (2009). A regulatory role of the Bateman domain of IMP dehydrogenase in adenylate nucleotide biosynthesis. J Biol Chem 284, 7960-7969.

Prosise, G. L., Wu, J. Z. \& Luecke, H. (2002). Crystal structure of Tritrichomonas foetus inosine monophosphate dehydrogenase in complex with the inhibitor ribavirin monophosphate reveals a 
catalysis-dependent ion-binding site. J Biol Chem 277, 5065450659.

Reddy, V. M., Einck, L. \& Nacy, C. A. (2008). In vitro antimycobacterial activities of capuramycin analogues. Antimicrob Agents Chemother 52, 719-721.

Risal, D., Strickler, M. D. \& Goldstein, B. M. (2004). Ternary complex of the human type II inosine monophosphate dehydrogenase with 6Cl-IMP and NAD. doi: 10.2210/pdb1nfb/pdb.

Sassetti, C. M., Boyd, D. H. \& Rubin, E. J. (2001). Comprehensive identification of conditionally essential genes in mycobacteria. Proc Natl Acad Sci U S A 98, 12712-12717.

Sassetti, C. M., Boyd, D. H. \& Rubin, E. J. (2003). Genes required for mycobacterial growth defined by high density mutagenesis. Mol Microbiol 48, 77-84.

Shu, Q. \& Nair, V. (2008). Inosine monophosphate dehydrogenase (IMPDH) as a target in drug discovery. Med Res Rev 28, 219-232.

Striepen, B., White, M. W., Li, C., Guerini, M. N., Malik, S. B., Logsdon, J. M., Jr, Liu, C. \& Abrahamsen, M. S. (2002). Genetic complementation in apicomplexan parasites. Proc Natl Acad Sci U S A 99, 63046309.

Tiedeman, A. A. \& Smith, J. M. (1985). Nucleotide sequence of the guaB locus encoding IMP dehydrogenase of Escherichia coli K12. Nucleic Acids Res 13, 1303-1316.
Umejiego, N. N., Li, C., Riera, T., Hedstrom, L. \& Striepen, B. (2004). Cryptosporidium parvum IMP dehydrogenase: identification of functional, structural, and dynamic properties that can be exploited for drug design. J Biol Chem 279, 40320-40327.

Verham, R., Meek, T. D., Hedstrom, L. \& Wang, C. C. (1987). Purification, characterization, and kinetic analysis of inosine $5^{\prime}$ monophosphate dehydrogenase of Tritrichomonas foetus. Mol Biochem Parasitol 24, 1-12.

Xiang, B., Taylor, J. C. \& Markham, G. D. (1996). Monovalent cation activation and kinetic mechanism of inosine $5^{\prime}$-monophosphate dehydrogenase. J Biol Chem 271, 1435-1440.

Zhang, R., Evans, G., Rotella, F. J., Westbrook, E. M., Beno, D., Huberman, E., Joachimiak, A. \& Collart, F. R. (1999). Characteristics and crystal structure of bacterial inosine-5'-monophosphate dehydrogenase. Biochemistry 38, 4691-4700.

Zhang, X. H., He, K. W., Duan, Z. T., Zhou, J. M., Yu, Z. Y., Ni, Y. X. \& Lu, C. P. (2009). Identification and characterization of inosine 5monophosphate dehydrogenase in Streptococcus suis type 2. Microb Pathog 47, 267-273.

Zhou, X., Cahoon, M., Rosa, P. \& Hedstrom, L. (1997). Expression, purification, and characterization of inosine $5^{\prime}$-monophosphate dehydrogenase from Borrelia burgdorferi. J Biol Chem 272, 2197721981.

Edited by: S. V. Gordon 